\title{
turboEELS - A code for the simulation of the electron energy loss and inelastic X-ray scattering spectra using the Liouville-Lanczos approach to time-dependent density-functional perturbation theory
}

\author{
Iurii Timrov ${ }^{\mathrm{a}, \mathrm{b}, 1}$, Nathalie Vast ${ }^{\mathrm{a}}$, Ralph Gebauer ${ }^{\mathrm{c}}$, Stefano Baroni ${ }^{\mathrm{b}, *}$ \\ ${ }^{a}$ Laboratoire des Solides Irradiés, École Polytechnique - CEA - DSM - IRAMIS - CNRS \\ UMR 7642, 91128 Palaiseau cedex, France \\ ${ }^{b}$ SISSA - Scuola Internazionale Superiore di Studi Avanzati, Via Bonomea 265, 34136 \\ Trieste, Italy \\ ${ }^{c}$ ICTP - The Abdus Salam International Centre for Theoretical Physics, Strada Costiera \\ 11, 34151 Trieste, Italy
}

\begin{abstract}
We introduce turboEELS, an implementation of the Liouville-Lanczos approach to linearized time-dependent density-functional theory, designed to simulate electron energy loss and inelastic X-ray scattering spectra in periodic solids. turboEELS is open-source software distributed under the terms of the GPL as a component of QUANTUM ESPRESSO. As with other components, turboEELS is optimized to run on a variety of different platforms, from laptops to massively parallel architectures, using native mathematical libraries (LAPACK and FFTW) and a hierarchy of custom parallelization layers built on top of MPI.

Keywords: Electron energy loss spectroscopy, Inelastic X-ray scattering, Time-dependent density functional perturbation theory, Quantum ESPRESSO, Linear response, Liouville-Lanczos approach

\section{PROGRAM SUMMARY}

Program Title: turboEELS

Catalogue identifier:

Program summary URL:

Program obtainable from: CPC Program Library, Queen's University, Belfast, N. Ireland

Licensing provisions: GNU General Public License V 2.0

No. of lines in distributed program, including test data, etc.: $15000+$ libraries

No. of bytes in distributed program, including test data, etc.: 6231803

\footnotetext{
*Corresponding author. e-mail address: baroni@sissa.it

${ }^{1}$ Present address: SISSA - Scuola Internazionale Superiore di Studi Avanzati, Via Bonomea 265, 34136 Trieste, Italy
} 
Distribution format: tar.gz

Programming language: Fortran 95

Computer: Any computer architecture

Operating system: GNU/Linux, AIX, IRIX, Mac OS X, and other UNIX-like OS's

Classification: 7.2

External routines: turboEELS is a tightly integrated component of the QUANTUM ESPRESSO distribution and requires the standard libraries linked by it: BLAS, LAPACK, FFTW, MPI.

Nature of problem: Calculation of the electron energy loss and inelastic X-ray scattering spectra of periodic solids.

Solution method: The charge-density susceptibility of a periodic system is expressed in terms of the resolvent of its Liouvillian superoperator within time-dependent density functional perturbation theory. It is calculated using non-Hermitian or pseudoHermitian variants of the Lanczos recursion scheme, whose implementation does not require the calculation of any virtual states. Pseudopotentials (both norm-conserving and ultrasoft) are used in conjunction with plane-wave basis sets and periodic boundary conditions. Relativistic effects (spin-orbit coupling) can be included in calculations.

Restrictions: Linear-response regime. Adiabatic exchange-correlation kernels only. No hybrid functionals. Collinear spin-polarized formalism is not supported, only noncollinear spin-polarized case can be used. Spin-orbit coupling cannot be used with ultrasoft pseudopotentials. No magnetism. No Hubbard U formalism. No PAW pseudopotentials.

Unusual features: No virtual orbitals are used, nor even calculated. A single Lanczos recursion gives access to the whole spectrum at fixed transferred momentum.

Additional comments: The distribution file of this program can be downloaded from the QUANTUM ESPRESSO website: http://www.quantum-espresso.org/, and the development version of this program can be downloaded via SVN from the QE-forge website: http://qe-forge.org/gf/project/q-e/ .

Running time: From a few minutes for elemental bulk systems with a few atoms in the primitive unit cell on serial machines up to many hours on multiple processors for complex systems (e.g., surfaces with high Miller indices) with dozens or hundreds of atoms.

\section{Introduction}

Electron energy loss (EEL) and inelastic X-ray scattering (IXS) spectroscopies are two experimental techniques that probe collective (plasmon-like) and single-particle charge excitations [1, 2] in extended systems. Nowadays, EEL experiments with transmission electron microscopes allow one to reach an extremely high spatial resolution of $\sim 0.1 \mathrm{~nm}$ and an energy resolution of typically $1 \mathrm{eV}$, which can be reduced down to $\sim 0.1 \mathrm{eV}$ if an electron-beam monochromator is used [3, 4, 5]. The EEL and IXS cross sections are directly related to the imaginary part of the inverse of the dielectric function, and thus many electronic properties of the probed materials can be determined. On the theoretical side, time-dependent (TD) density functional theory (DFT) [6, 7, 8] 
is the state-of-the-art method that allows one to gain access to collective and single-particle excitations, resulting in an optimal compromise between computational cost and accuracy 9 .

In the "conventional" TDDFT approach to EEL and IXS spectroscopies, the information about plasmon and single-particle excitations is obtained from the poles of the charge-density susceptibility, by first computing the independentelectron susceptibility and solving then a Dyson-like screening equation for the full response function. However, such an approach has several drawbacks: i) the calculation of the independent-electron susceptibility requires the knowledge of numerous empty states, which are hardly available for systems larger than a few handfuls of independent atoms; ii) the solution of the Dyson-like screening equation requires multiple time-consuming inversions and multiplications of large matrices; and iii) the first two steps must be repeated for every value of frequency one is interested in, making it difficult to study extended frequency ranges.

Recently a new technique was proposed to overcome these drawbacks, based on linearized TDDFT (Time-Dependent Density Functional Perturbation Theory, TDDFpT) [10, 11, 12, 13, and inspired by the Liouville-Lanczos (LL) approach to optical spectroscopies in molecular systems, which is in turn the extension to the dynamical regime of time-independent density-functional perturbation theory (DFPT) [14, 15. The distinctive feature of the new approach is that it allows for the full spectrum of a periodic solid to be computed over a broad frequency range, with a computational effort that is only a few times larger than that needed by a single ground-state DFT or static DFPT calculation at any given finite transferred momentum.

In this paper we introduce a computer code, named turboEELS, which implements the LL approach to EEL and IXS spectroscopies within TDDFpT. turboEELS has a similar structure as the turboTDDFT code used to compute absorption spectra in molecular systems [16, 17, and these two codes share indeed a large number of routines (in addition, turboEELS also leverages a certain number of solid-state specific routines from the ph.x component of QUANTUM ESPRESSO). turboEELS is distributed under the terms of the GPL license [18, as a component of the QUANTUM ESPRESSO suite of open-source codes based on plane-wave basis sets, pseudopotentials, and using periodic boundary conditions 19 .

This paper is organized as follows. In Sec. 2 we provide a theoretical background for the LL approach to EEL and IXS spectroscopies. In Sec. 3 we describe the components of turboEELS which is a part of the QUANTUM ESPRESSO distribution. In Sec. 4 we provide the instructions for installing turboEELS on UNIX systems and discuss various levels of parallelization implemented in it. In Sec. 5 we give an example of the usage of turboEELS for the calculation of the EEL spectra of diamond. Finally, Sec. 6 contains conclusions and perspectives for future work. 


\section{Theory}

\subsection{Statement of the problem}

Both EEL and IXS spectroscopies probe the electronic structure of the materials [20]. In an EEL experiment, an electron with wave vector $\mathbf{k}_{i}$ and energy $E_{i}=\frac{\hbar^{2} k_{i}^{2}}{2 m}$ undergoes an inelastic scattering with the electrons and ions of the target sample, which modifies the electronic wave vector and energy to $\mathbf{k}_{f}=\mathbf{k}_{i}-\mathbf{Q}$ and $E_{f}=E_{i}-\hbar d \omega$, where $\hbar \mathbf{Q}$ and $\hbar d \omega$ are the momentum and energy transferred to the sample, respectively. Analogously, in an IXS experiment an X-ray photon of energy $E_{i}=\hbar \omega_{i}$ and polarization $\mathbf{e}_{i}$ is scattered to a final state of energy $E_{f}=\hbar \omega_{f}$ and polarization $\mathbf{e}_{f}$. The corresponding double-differential cross section reads [1, 2]:

$$
\frac{d^{2} \sigma}{d \Omega d \omega}=A S(\mathbf{Q}, \omega)
$$

where $S(\mathbf{Q}, \omega)$ is the dynamic structure factor (per unit volume) of the target sample and

$$
\begin{aligned}
A_{\mathrm{EEL}} & =\left(\frac{4 \pi e^{2}}{Q^{2}}\right)^{2} \frac{m^{2}}{4 \pi^{2} \hbar^{4}} \frac{k_{f}}{k_{i}}, \\
A_{\mathrm{IXS}} & =\left(\frac{e^{2}}{m c^{2}}\right)^{2}\left(\mathbf{e}_{i} \cdot \mathbf{e}_{f}\right)^{2} \frac{\omega_{f}}{\omega_{i}},
\end{aligned}
$$

is the probe factor, whose form differs for the two spectroscopies. In the above expressions, $-e$ and $m$ are the electron charge and mass, respectively, and $c$ is the speed of light.

According to the fluctuation-dissipation theorem the dynamic structure factor, which describes charge-density fluctuations in the system, is related to the imaginary part of the charge-density susceptibility, $\chi\left(\mathbf{Q}, \mathbf{Q}^{\prime} ; \omega\right)$, which describes energy dissipation, by the relation [21]:

$$
S(\mathbf{Q}, \omega)=-\frac{\hbar}{\pi} \operatorname{Im} \chi(\mathbf{Q}, \mathbf{Q} ; \omega) .
$$

The poles of $\chi(\mathbf{Q}, \mathbf{Q} ; \omega)$ correspond to plasmon frequencies and single-particle excitations.

In periodic solids the transferred momentum, $\mathbf{Q}$, can be expressed as the sum of an irreducible component lying inside of the first Brillouin zone (BZ), q, and a reciprocal lattice vector, $\mathbf{G}: \mathbf{Q}=\mathbf{q}+\mathbf{G}$. The EEL/IXS cross section is often expressed in terms of the inverse dielectric matrix, $\epsilon_{\mathbf{G G}^{\prime}}^{-1}(\mathbf{q} ; \omega) \equiv \epsilon^{-1}(\mathbf{q}+$ $\left.\mathbf{G}, \mathbf{q}+\mathbf{G}^{\prime} ; \omega\right)$, defined as $[22,23]$ :

$$
\epsilon^{-1}\left(\mathbf{q}+\mathbf{G}, \mathbf{q}+\mathbf{G}^{\prime} ; \omega\right)=\delta_{\mathbf{G G}^{\prime}}+\frac{4 \pi e^{2}}{|\mathbf{q}+\mathbf{G}|^{2}} \chi\left(\mathbf{q}+\mathbf{G}, \mathbf{q}+\mathbf{G}^{\prime} ; \omega\right) .
$$

The diagonal elements of the inverse dielectric matrix define the inverse dielectric function, $\epsilon^{-1}(\mathbf{Q}, \omega) \equiv \epsilon^{-1}(\mathbf{q}+\mathbf{G}, \mathbf{q}+\mathbf{G} ; \omega)$, whose imaginary part is the 
negative of the so-called loss function, and which satisfies the Thomas-ReicheKuhn (or $f$-sum) rule [24]:

$$
\int_{0}^{\infty} \operatorname{Im}\left[\epsilon^{-1}(\mathbf{Q}, \omega)\right] \omega d \omega=-\frac{\pi}{2} \omega_{p}^{2},
$$

where $\omega_{p}=\sqrt{4 \pi e^{2} n / m}$ is the Drude plasma frequency, where $n$ is the average electron density. The dielectric function is defined as the inverse of the inverse dielectric function, $\epsilon(\mathbf{Q}, \omega) \equiv 1 / \epsilon^{-1}(\mathbf{Q}, \omega)$ (Note: this is not a matrix inversion). The information about the real and imaginary parts of $\epsilon(\mathbf{Q}, \omega)$ can be very useful for an analysis of the EEL/IXS spectra (i.e. the loss function), because the loss function, $-\operatorname{Im}\left[\epsilon^{-1}(\mathbf{Q}, \omega)\right]=\operatorname{Im}[\epsilon(\mathbf{Q}, \omega)] /\left(\operatorname{Re}[\epsilon(\mathbf{Q}, \omega)]^{2}+\operatorname{Im}[\epsilon(\mathbf{Q}, \omega)]^{2}\right)$, has peaks when $\operatorname{Re}[\epsilon(\mathbf{Q}, \omega)]=0$ and $\operatorname{Im}[\epsilon(\mathbf{Q}, \omega)]$ is small. For small $\mathbf{Q}$ the plasmon occurs when $\operatorname{Re}[\epsilon(\mathbf{Q}, \omega)]=0$ and it has a positive slope (changes the sign from negative to positive).

\subsection{Quantum Liouville equation for the charge-density susceptibility}

As we have seen in the previous section, the EEL/IXS cross section is essentially determined by the diagonal of the charge-density susceptibility, $\chi(\mathbf{Q}, \mathbf{Q} ; \omega)$. The latter quantity is the response of the Fourier transform of the charge-density operator, $\hat{n}_{\mathbf{Q}} \rightarrow \mathrm{e}^{\mathbf{i} \cdot \mathbf{r}}$, to a monochromatic perturbation of same wave vector, $\hat{v}_{\text {ext }, \mathbf{q}}^{\prime}=\hat{n}_{\mathbf{Q}}$. Here and in the following quantum-mechanical operators and superoperators are denoted by a hat "^", whereas their coordinate representation does not have any hat. Here $\mathbf{q}$ denotes the irreducible component of $\mathbf{Q}$ and the notation has been chosen so as to emphasize that the response to a monochromatic perturbation is in general not monochromatic, while conserving the irreducible component of its wave vector. In particular, the response of the density matrix to a monochromatic perturbation, $\hat{v}_{\text {ext, } \mathbf{q}}^{\prime}=\hat{n}_{\mathbf{Q}}$, can be labeled by the irreducible component of its wave vector, $\hat{\rho}_{\mathbf{q}}^{\prime}$, and so can the response of the Hartree and exchange-correlation (HXC) potential, $\hat{v}_{\mathrm{HXC}, \mathbf{q}}^{\prime}\left[\hat{\rho}_{\mathbf{q}}^{\prime}\right]$ (i.e. they result to be the product of a lattice-periodic function times a plane

wave with wave vector $\mathbf{q}, \mathrm{e}^{i \mathbf{q} \cdot \mathbf{r}}$ ). In terms of $\hat{\rho}_{\mathbf{q}}^{\prime}$, the relevant matrix element of the charge-density susceptibility reads:

$$
\chi(\mathbf{Q}, \mathbf{Q} ; \omega)=\operatorname{Tr}\left(\hat{n}_{\mathbf{Q}}^{\dagger} \hat{\rho}_{\mathbf{q}}^{\prime}\right) .
$$

As it was shown in Refs. [12, 13, 25, the response density matrix satisfies a superoperator linear equation - the quantum Liouville equation - that can be symbolically cast as:

$$
(\omega-\hat{\mathcal{L}}) \cdot \hat{\rho}_{\mathbf{q}}^{\prime}(\omega)=\left[\hat{v}_{\text {ext }, \mathbf{q}}^{\prime}, \hat{\rho}^{\circ}\right],
$$

where the action of the Liouvillian superoperator $\hat{\mathcal{L}}$ onto $\hat{\rho}_{\mathbf{q}}^{\prime}$ is defined as:

$$
\hat{\mathcal{L}} \cdot \hat{\rho}_{\mathbf{q}}^{\prime} \equiv\left[\hat{H}^{\circ}, \hat{\rho}_{\mathbf{q}}^{\prime}\right]+\left[\hat{v}_{\mathrm{HXC}, \mathbf{q}}^{\prime}\left[\hat{\rho}_{\mathbf{q}}^{\prime}\right], \hat{\rho}^{\circ}\right]
$$


and Hartree atomic units $(e=m=\hbar=1)$ are used henceforth. Here $[\cdot, \cdot]$ indicates a quantum commutator, $\hat{H}^{\circ}$ and $\hat{\rho}^{\circ}$ are the unperturbed Kohn-Sham (KS) Hamiltonian and density matrix, respectively. According to the above considerations, the relevant matrix element of the charge-density susceptibility can be represented as a suitable off-diagonal matrix element of the resolvent of the Liouvillian superoperator as:

$$
\chi(\mathbf{Q}, \mathbf{Q} ; \omega)=\left(\hat{n}_{\mathbf{Q}},(\omega-\hat{\mathcal{L}})^{-1} \cdot\left[\hat{n}_{\mathbf{Q}}, \hat{\rho}^{\circ}\right]\right) .
$$

The essential ingredients for evaluating Eq. (10) are thus an explicit representation for the response density matrix and an efficient way of evaluating off-diagonal matrix elements of the resolvent of linear (super-) operators. The subsections 2.4 and 2.5 are devoted to these two tasks, respectively. The problem of computing the charge-density susceptibility (10) is mapped onto the solution of a set of coupled linearized KS equations in the self-consistent way, as will be shown in Sec. 2.3.

\subsection{Bloch functions and coordinate representation of (response) operators}

In periodic solids the unperturbed KS orbitals, which satisfy the groundstate KS equations, have a Bloch form: $\varphi_{n, \mathbf{k}}^{\circ}(\mathbf{r})=\mathrm{e}^{i \mathbf{k} \cdot \mathbf{r}} \mathrm{u}_{n, \mathbf{k}}^{\circ}(\mathbf{r})$, where $n$ is a band index, $\mathbf{k}$ is a point in BZ, and $u_{n, \mathbf{k}}^{\circ}(\mathbf{r})$ is a lattice-periodic function. The response KS orbitals corresponding to each monochromatic $\mathbf{q}$ component of the perturbing potential, $\hat{v}_{\text {ext }, \mathbf{q}}^{\prime}$, read: $\tilde{\varphi}_{n, \mathbf{k}+\mathbf{q}}^{\prime}(\mathbf{r}, \omega)=\mathrm{e}^{i(\mathbf{k}+\mathbf{q}) \cdot \mathbf{r}} \tilde{\mathrm{u}}_{n, \mathbf{k}+\mathbf{q}}^{\prime}(\mathbf{r}, \omega)$, where $\tilde{\mathbf{u}}_{n, \mathbf{k}+\mathbf{q}}^{\prime}(\mathbf{r}, \omega)$ is the lattice-periodic response orbital. The response and perturbing potentials in the coordinate representation $\operatorname{read}$ as $\tilde{v}_{\mathbf{q}}^{\prime}(\mathbf{r}, \omega)=\mathrm{e}^{i \mathbf{q} \cdot \mathbf{r}} \tilde{\mathbf{v}}_{\mathbf{q}}^{\prime}(\mathbf{r}, \omega)$, where $\tilde{\mathbf{v}}_{\mathbf{q}}^{\prime}(\mathbf{r}, \omega)$ is the lattice-periodic part of the potential. Here and below, Roman letters in formulas indicate lattice-periodic parts of Bloch functions, and tildes " " indicate Fourier transforms of functions in the frequency domain.

It can be demonstrated that the lattice-periodic response orbitals, $\tilde{\mathbf{u}}_{n, \mathbf{k}+\mathbf{q}}^{\prime}(\mathbf{r}, \omega)$, satisfy the linearized and Fourier transformed KS equations [10]:

$$
\begin{aligned}
\left(\hat{\mathrm{H}}_{\mathbf{k}+\mathbf{q}}^{\circ}-\varepsilon_{n, \mathbf{k}}^{\circ}-\omega\right) \tilde{\mathrm{u}}_{n, \mathbf{k}+\mathbf{q}}^{\prime}(\mathbf{r}, \omega) & +\hat{\mathrm{P}}_{c}^{\mathbf{k}+\mathbf{q}} \tilde{\mathrm{v}}_{\mathrm{HXC}, \mathbf{q}}^{\prime}(\mathbf{r}, \omega) \mathrm{u}_{n, \mathbf{k}}^{\circ}(\mathbf{r}) \\
& =-\hat{\mathrm{P}}_{c}^{\mathbf{k}+\mathbf{q}} \tilde{\mathrm{v}}_{\mathrm{ext}, \mathbf{q}}^{\prime}(\mathbf{r}, \omega) \mathrm{u}_{n, \mathbf{k}}^{\circ}(\mathbf{r}),
\end{aligned}
$$

where $\varepsilon_{n, \mathbf{k}}^{\circ}$ are the ground-state KS energies, $\tilde{\mathrm{v}}_{\text {ext, }}^{\prime}(\mathbf{r}, \omega)=\mathrm{e}^{i \mathbf{G} \cdot \mathbf{r}}$ is the latticeperiodic part of the external perturbing potential $\tilde{v}_{\text {ext }, \mathbf{q}}^{\prime}(\mathbf{r}, \omega)$, and $\tilde{\mathrm{v}}_{\mathrm{HXC}, \mathbf{q}}^{\prime}$ is the lattice-periodic q component of the HXC response potential, which reads:

$$
\tilde{\mathrm{v}}_{\mathrm{HXC}, \mathbf{q}}^{\prime}(\mathbf{r}, \omega)=\int \kappa\left(\mathbf{r}, \mathbf{r}^{\prime}\right) \tilde{\mathrm{n}}_{\mathbf{q}}^{\prime}\left(\mathbf{r}^{\prime}, \omega\right) \mathrm{e}^{-i \mathbf{q} \cdot\left(\mathbf{r}-\mathbf{r}^{\prime}\right)} d \mathbf{r}^{\prime},
$$

where $\kappa\left(\mathbf{r}, \mathbf{r}^{\prime}\right)=1 /\left|\mathbf{r}-\mathbf{r}^{\prime}\right|+\kappa_{\mathrm{XC}}\left(\mathbf{r}, \mathbf{r}^{\prime}\right), \kappa_{\mathrm{XC}}\left(\mathbf{r}, \mathbf{r}^{\prime}\right)$ is the XC kernel [26], which in the adiabatic DFT approximation adopted in this paper is independent of frequency, and $\tilde{n}_{\mathbf{q}}^{\prime}(\mathbf{r}, \omega)$ is the lattice-periodic $\mathbf{q}$ component of the response chargedensity. In practice, the response $\mathrm{XC}$ potential is computed in the real space, 
whereas the response Hartree potential is computed in the reciprocal space as:

$$
\tilde{\mathrm{v}}_{\mathrm{H}, \mathbf{q}}^{\prime}(\mathbf{r}, \omega)=4 \pi \sum_{\mathbf{G}} \frac{\tilde{\mathrm{n}}_{\mathbf{q}}^{\prime}(\mathbf{G}, \omega)}{|\mathbf{q}+\mathbf{G}|^{2}} \mathrm{e}^{i \mathbf{G} \cdot \mathbf{r}} .
$$

In Eq. (11), as well as in the rest of this paper, quantum-mechanical operators having a wave vector subscript ( $\operatorname{such}$ as $\hat{\mathrm{H}}_{\mathbf{k}+\mathbf{q}}^{\circ}$ ) or superscript (such as $\hat{\mathrm{P}}_{c}^{\mathbf{k}+\mathbf{q}}$ ) operate on lattice-periodic functions, and they are defined in terms of their coordinate representations as:

$$
\begin{aligned}
H^{\circ}\left(\mathbf{r}, \mathbf{r}^{\prime}\right) & =\sum_{\mathbf{k}} \mathrm{e}^{i \mathbf{k} \cdot\left(\mathbf{r}-\mathbf{r}^{\prime}\right)} \mathrm{H}_{\mathbf{k}}^{\circ}\left(\mathbf{r}, \mathbf{r}^{\prime}\right), \\
P_{c}\left(\mathbf{r}, \mathbf{r}^{\prime}\right) & =\sum_{\mathbf{k}} \mathrm{e}^{i \mathbf{k} \cdot\left(\mathbf{r}-\mathbf{r}^{\prime}\right)} \mathrm{P}_{c}^{\mathbf{k}}\left(\mathbf{r}, \mathbf{r}^{\prime}\right),
\end{aligned}
$$

where the projector onto the conduction manifold, $\mathrm{P}_{c}^{\mathbf{k}}\left(\mathbf{r}, \mathbf{r}^{\prime}\right)$, can be expressed in terms of the lattice-periodic parts of the unperturbed Bloch functions as:

$$
\mathrm{P}_{c}^{\mathbf{k}}\left(\mathbf{r}, \mathbf{r}^{\prime}\right)=\delta\left(\mathbf{r}-\mathbf{r}^{\prime}\right)-\sum_{n}^{o c c} \mathrm{u}_{n, \mathbf{k}}^{\circ}(\mathbf{r}) \mathrm{u}_{n, \mathbf{k}}^{\circ *}\left(\mathbf{r}^{\prime}\right),
$$

where the sum extends over all the occupied bands. Thus, in Eq. 111 no empty states are needed to determine the response orbitals, much in the same way like in static density functional perturbation theory (DFPT) [14, 15].

The monochromatic q component of the response density matrix in the coordinate representation reads:

$$
\begin{aligned}
\tilde{\rho}_{\mathbf{q}}^{\prime}\left(\mathbf{r}, \mathbf{r}^{\prime} ; \omega\right)= & 2 \sum_{n, \mathbf{k}}\left(\tilde{\varphi}_{n, \mathbf{k}+\mathbf{q}}^{\prime}(\mathbf{r}, \omega) \varphi_{n, \mathbf{k}}^{\circ *}\left(\mathbf{r}^{\prime}\right)+\varphi_{n, \mathbf{k}}^{\circ *}(\mathbf{r}) \tilde{\varphi}_{n,-\mathbf{k}-\mathbf{q}}^{\prime *}\left(\mathbf{r}^{\prime},-\omega\right)\right) \\
= & 2 \sum_{n, \mathbf{k}}\left(\mathrm{e}^{i \mathbf{q} \cdot \mathbf{r}} \tilde{\mathbf{u}}_{n, \mathbf{k}+\mathbf{q}}^{\prime}(\mathbf{r}, \omega) \mathrm{u}_{n, \mathbf{k}}^{\circ *}\left(\mathbf{r}^{\prime}\right) \mathrm{e}^{i \mathbf{k} \cdot\left(\mathbf{r}-\mathbf{r}^{\prime}\right)}\right. \\
& \left.\quad+\mathrm{e}^{i \mathbf{q} \cdot \mathbf{r}^{\prime}} \mathrm{u}_{n, \mathbf{k}}^{\circ *}(\mathbf{r}) \tilde{\mathbf{u}}_{n,-\mathbf{k}-\mathbf{q}}^{\prime *}\left(\mathbf{r}^{\prime},-\omega\right) \mathrm{e}^{-i \mathbf{k} \cdot\left(\mathbf{r}-\mathbf{r}^{\prime}\right)}\right),
\end{aligned}
$$

where the factor of 2 is due to spin degeneracy. Hence, the response chargedensity, which is a diagonal of the response density matrix, $\tilde{n}_{\mathbf{q}}^{\prime}(\mathbf{r}, \omega)=\tilde{\rho}_{\mathbf{q}}^{\prime}(\mathbf{r}, \mathbf{r} ; \omega)$, reads:

$$
\tilde{n}_{\mathbf{q}}^{\prime}(\mathbf{r}, \omega)=\mathrm{e}^{i \mathbf{q} \cdot \mathbf{r}} \tilde{\mathrm{n}}_{\mathbf{q}}^{\prime}(\mathbf{r}, \omega)
$$

where $\tilde{\mathbf{n}}_{\mathbf{q}}^{\prime}(\mathbf{r}, \omega)$ is the lattice-periodic charge-density, which reads:

$$
\tilde{\mathrm{n}}_{\mathbf{q}}^{\prime}(\mathbf{r}, \omega)=2 \sum_{n, \mathbf{k}} \mathrm{u}_{n, \mathbf{k}}^{\circ *}(\mathbf{r})\left(\tilde{\mathrm{u}}_{n, \mathbf{k}+\mathbf{q}}^{\prime}(\mathbf{r}, \omega)+\tilde{\mathrm{u}}_{n,-\mathbf{k}-\mathbf{q}}^{\prime *}(\mathbf{r},-\omega)\right) .
$$

The antiresonant contribution to the response density matrix 17) satisfies the equation:

$$
\begin{aligned}
\left(\hat{\mathrm{H}}_{\mathbf{k}+\mathbf{q}}^{\circ}-\varepsilon_{n, \mathbf{k}}^{\circ}+\omega\right) \tilde{\mathrm{u}}_{n,-\mathbf{k}-\mathbf{q}}^{\prime *}(\mathbf{r},-\omega) & +\hat{\mathrm{P}}_{c}^{\mathbf{k}+\mathbf{q}} \tilde{\mathrm{v}}_{\mathrm{HXC}, \mathbf{q}}^{\prime}(\mathbf{r}, \omega) \mathrm{u}_{n, \mathbf{k}}^{\circ}(\mathbf{r}) \\
& =-\hat{\mathrm{P}}_{c}^{\mathbf{k}+\mathbf{q}} \tilde{\mathrm{v}}_{\mathrm{ext}, \mathbf{q}}^{\prime}(\mathbf{r}, \omega) \mathrm{u}_{n, \mathbf{k}}^{\circ}(\mathbf{r}),
\end{aligned}
$$


which can be obtained from Eq. (11) by complex conjugation and simple manipulations deriving from time-reversal invariance of the unperturbed system $\left[\mathrm{u}_{n, \mathbf{k}}^{\circ}(\mathbf{r})=\mathrm{u}_{n,-\mathbf{k}}^{\circ *}(\mathbf{r})\right]$ and the reality of the perturbing and HXC response potentials $\left(\tilde{\mathrm{v}}_{\mathrm{ext}, \mathbf{q}}^{\prime}(\mathbf{r}, \omega)=\tilde{\mathrm{v}}_{\mathrm{ext},-\mathbf{q}}^{\prime *}(\mathbf{r},-\omega)\right.$ and $\tilde{\mathrm{v}}_{\mathrm{HXC}, \mathbf{q}}^{\prime}(\mathbf{r}, \omega)=\tilde{\mathrm{v}}_{\mathrm{HXC},-\mathbf{q}}^{\prime *}(\mathbf{r},-\omega)$, respectively). The set of coupled KS equations (11) and (20) represent the quantum Liouville equation (8).

\subsection{Standard batch representation of operators}

Equations (11), (17) and (20) show that the response of the charge-density of a periodic solid to a perturbation of wave vector $\mathbf{Q}$ is uniquely determined by the two sets of response orbitals $\left\{\tilde{\mathbf{u}}_{n, \mathbf{k}+\mathbf{q}}^{\prime}(\mathbf{r}, \omega)\right\}$ and $\left\{\tilde{\mathbf{u}}_{n,-\mathbf{k}-\mathbf{q}}^{\prime *}(\mathbf{r},-\omega)\right\}$. Note that $n$ and $\mathbf{k}$ are running indices, whereas $\mathbf{q}$ is fixed. It is convenient to consider a linear combination of these functions, defined as

$$
\begin{aligned}
& \mathrm{q}_{n, \mathbf{k}+\mathbf{q}}(\mathbf{r})=\frac{1}{2}\left(\tilde{\mathrm{u}}_{n, \mathbf{k}+\mathbf{q}}^{\prime}(\mathbf{r}, \omega)+\tilde{\mathrm{u}}_{n,-\mathbf{k}-\mathbf{q}}^{\prime *}(\mathbf{r},-\omega)\right), \\
& \mathrm{p}_{n, \mathbf{k}+\mathbf{q}}(\mathbf{r})=\frac{1}{2}\left(\tilde{\mathrm{u}}_{n, \mathbf{k}+\mathbf{q}}^{\prime}(\mathbf{r}, \omega)-\tilde{\mathrm{u}}_{n,-\mathbf{k}-\mathbf{q}}^{\prime *}(\mathbf{r},-\omega)\right) .
\end{aligned}
$$

The two sets of response orbitals $\mathrm{q}_{\mathbf{q}}=\left\{\mathrm{q}_{n, \mathbf{k}+\mathbf{q}}\right\}$ and $\mathrm{p}_{\mathbf{q}}=\left\{\mathrm{p}_{n, \mathbf{k}+\mathbf{q}}\right\}$ are called, respectively, the upper and lower components of the standard batch representation (SBR) [13, 25] of the response density matrix supervector: $\hat{\rho}_{\mathbf{q}}^{\prime} \stackrel{\mathrm{SBR}}{\longrightarrow}\left\{\mathrm{q}_{\mathbf{q}}, \mathrm{p}_{\mathbf{q}}\right\}$ [16. In the SBR the set of Eqs. (11) and 20) can be cast into the compact form:

$$
\left(\omega \hat{I}-\hat{\mathcal{L}}_{\mathbf{q}}\right)\left(\begin{array}{c}
\mathrm{q}_{\mathbf{q}} \\
\mathrm{p}_{\mathbf{q}}
\end{array}\right)=\left(\begin{array}{c}
0 \\
\mathrm{y}_{\mathbf{q}}
\end{array}\right),
$$

where $\hat{I}$ is the unit matrix, and the Liouvillian $\hat{\mathcal{L}}_{\mathbf{q}}$ has the block form [13, 25]:

$$
\hat{\mathcal{L}}_{\mathbf{q}}=\left(\begin{array}{cc}
0 & \hat{\mathcal{D}}_{\mathbf{q}} \\
\hat{\mathcal{D}}_{\mathbf{q}}+\hat{\mathcal{K}}_{\mathbf{q}} & 0
\end{array}\right)
$$

where $\hat{\mathcal{D}}_{\mathbf{q}}$ and $\hat{\mathcal{K}}_{\mathbf{q}}$ superoperators are defined by their action on batches,

$$
\begin{aligned}
& \hat{\mathcal{D}}_{\mathbf{q}} \mathrm{q}_{\mathbf{q}}=\left\{\left(\hat{\mathrm{H}}_{\mathbf{k}+\mathbf{q}}^{\circ}-\varepsilon_{n, \mathbf{k}}^{\circ}\right) \mathrm{q}_{n, \mathbf{k}+\mathbf{q}}(\mathbf{r})\right\}, \\
& \hat{\mathcal{K}}_{\mathbf{q}} \mathbf{q}_{\mathbf{q}}=\left\{\hat{\mathrm{P}}_{c}^{\mathbf{k}+\mathbf{q}} \tilde{\mathrm{v}}_{\mathrm{HXC}, \mathbf{q}}^{\prime}(\mathbf{r}) \mathrm{u}_{n, \mathbf{k}}^{\circ}(\mathbf{r})\right\},
\end{aligned}
$$

and $\tilde{\mathrm{v}}_{\mathrm{HXC}, \mathbf{q}}^{\prime}$ is given by Eq. 12, and the lattice-periodic response charge-density reads (see Eq. 19)):

$$
\tilde{\mathrm{n}}_{\mathbf{q}}^{\prime}(\mathbf{r})=4 \sum_{n, \mathbf{k}} \mathrm{u}_{n, \mathbf{k}}^{\circ *}(\mathbf{r}) \mathrm{q}_{n, \mathbf{k}+\mathbf{q}}(\mathbf{r})
$$

and

$$
\mathrm{y}_{\mathbf{q}}=\left\{\hat{\mathrm{P}}_{c}^{\mathbf{k}+\mathbf{q}} \tilde{\mathrm{v}}_{\mathrm{ext}, \mathbf{q}}^{\prime}(\mathbf{r}) \mathrm{u}_{n, \mathbf{k}}^{\circ}(\mathbf{r})\right\} .
$$


According to the above equations, operating with the Liouvillian on a test supervector essentially requires a calculation of the response HXC potential, its application to each valence KS orbital, as well as the operation of the unperturbed Hamiltonian onto twice the number of valence KS states.

The SBR of the charge-density operator reads: $\hat{n}_{\mathbf{Q}} \stackrel{\mathrm{SBR}}{\longrightarrow}\left\{\left\{\hat{\mathrm{P}}_{c}^{\mathbf{k}+\mathbf{q}} \mathrm{e}^{i \mathbf{G} \cdot \mathbf{r}} \mathrm{u}_{n, \mathbf{k}}^{\circ}\right\}, 0\right\}$, and hence the SBR of the charge-density susceptibility, Eq. 10), reads:

$$
\chi(\mathbf{Q}, \mathbf{Q} ; \omega)=\left(\left\{\left\{\hat{\mathrm{P}}_{c}^{\mathbf{k}+\mathbf{q}} \mathrm{e}^{i \mathbf{G} \cdot \mathbf{r}} \mathrm{u}_{n, \mathbf{k}}^{\circ}\right\}, 0\right\},\left\{\mathrm{q}_{\mathbf{q}}, \mathrm{p}_{\mathbf{q}}\right\}\right),
$$

where $\left\{q_{\mathbf{q}}, p_{\mathbf{q}}\right\}$ is the solution of the quantum Liouville equation in the SBR (23). In practice, the charge-density susceptibility $(29)$ is computed using the Lanczos recursion algorithm, which is briefly discussed in the next subsection.

\subsection{Lanczos recursion algorithm}

From Eq. (10) it is seen that in order to compute the charge-density susceptibility, $\chi(\mathbf{Q}, \mathbf{Q} ; \omega)$, one needs to evaluate the off-diagonal matrix element of the resolvent of the Liouvillian, $\left(\omega-\hat{\mathcal{L}}_{\mathbf{q}}\right)^{-1}$. A straightforward inversion of such a matrix for big systems in plane-wave framework is a formidable task. However, there exists an efficient recursive algorithm, so-called Lanczos algorithm, which does not rely on the inversion of the matrices, but a recursive evaluation of an off-diagonal matrix element as in Eq. (10) 27]. We will briefly review two flavors of the Lanczos algorithm which can be used to compute the charge-density susceptibility, namely, non-Hermitian Lanczos biorthogonalization algorithm [13, 16, 25], and pseudo-Hermitian Lanczos algorithm [17, 28, 29]. A detailed description of the algorithms can be found in the corresponding references.

In the non-Hermitian Lanczos biorthogonalization algorithm, by starting from the initial pair of Lanczos vectors $u_{1}=v_{1}=\left\{0, \mathrm{y}_{\mathbf{q}}\right\}$ (see Eq. (28)), two coupled Lanczos chains are generated (because there are two coupled KS equations, resonant and antiresonant [10]) by recursively applying $\hat{\mathcal{L}}_{\mathbf{q}}$ and $\hat{\mathcal{L}}_{\mathbf{q}}^{\dagger}$ to the previous Lanczos chain vectors, $u_{i}$ and $v_{i}$ [10, 13]. A pair of biorthogonal basis sets of increasing dimension are thus recursively constructed, $\left\{u_{i}\right\}$ and $\left\{v_{i}\right\}$, where $i=\overline{1, M}$, and $M$ being the number of Lanczos iterations, and the Lanczos coefficients, $\beta_{i}$ and $\gamma_{i}$, are thus generated form a sparse $M$-dimensional tridiagonal matrix, ${ }^{M} T_{\mathbf{q}}$, which is nothing but an oblique projection of the Liouvillian onto such biorthogonal bases: $\left({ }^{M} T_{\mathbf{q}}\right)_{i j}=\left(u_{i}, \hat{\mathcal{L}}_{\mathbf{q}} v_{j}\right)$.

The pseudo-Hermitian Lanczos algorithm is two times faster than the nonHermitian Lanczos biorthogonalization algorithm, because the former algorithm requires twice as less operations (action of the Liouvillian, $\hat{\mathcal{L}}_{\mathbf{q}}$, on the Lanczos vectors) [17. The main idea is to define a metric of the linear space, which redefines the scalar products, and thus allows one to use a generalized Hermitian Lanczos algorithm. As a result of the use of this algorithm one also generates the tridiagonal matrix ${ }^{M} T_{\mathbf{q}}$.

After a generation of the tridiagonal matrix, ${ }^{M} T_{\mathbf{q}}$, the charge-density susceptibility (see Eqs. (10) and (29p) can be computed as [10]:

$$
\chi(\mathbf{Q}, \mathbf{Q} ; \omega) \simeq\left({ }^{M} z_{\mathbf{q}},\left(\omega^{M} I-{ }^{M} T_{\mathbf{q}}\right)^{-1} \cdot{ }^{M} e_{1}\right),
$$


where ${ }^{M} I$ is the $M$-dimensional unit matrix, ${ }^{M} e_{1}=\{1,0, \ldots, 0\}$ is the $M$ dimensional unit vector, and ${ }^{M} z_{\mathbf{q}}=\left(z_{1, \mathbf{q}}, z_{2, \mathbf{q}}, \ldots, z_{M, \mathbf{q}}\right)$ is the $M$-dimensional array whose coefficients $z_{j, \mathbf{q}}$ are computed on-the-fly of the Lanczos recursion and defined as:

$$
z_{j, \mathbf{q}}=\left(\left\{\left\{\hat{\mathrm{P}}_{c}^{\mathbf{k}+\mathbf{q}} \mathrm{e}^{i \mathbf{G} \cdot \mathbf{r}} \mathrm{u}_{n, \mathbf{k}}^{\circ}(\mathbf{r})\right\}, 0\right\}, v_{j}\right) .
$$

In practice, the right-hand side of Eq. (30) is computed by solving, for any given value of frequency, $\omega$, the equation:

$$
\left(\omega^{M} I-{ }^{M} T_{\mathbf{q}}\right){ }^{M} x_{\mathbf{q}}={ }^{M} e_{1},
$$

and calculating the scalar product afterwards:

$$
\chi(\mathbf{Q}, \mathbf{Q} ; \omega)=\left({ }^{M} z_{\mathbf{q}},{ }^{M} x_{\mathbf{q}}\right),
$$

which are both extremely inexpensive operations from the computational point of view. This allows one to study a wide frequency range for EEL and IXS. The convergence of the EEL/IXS spectra with respect to the number of Lanczos iterations, $M$, can be sped up by making use of the extrapolation technique for the Lanczos coefficients, which is described in detail in Refs. 13, 16.

\subsection{Extensions of the Liouville-Lanczos approach}

The LL formalism presented above has been generalized in several ways: i) metallic systems; ii) explicit account of symmetry for k-point sampling; iii) relativistic effects (spin-orbit coupling). A detailed discussion about these extensions can be found in Ref. [11, and here we discuss briefly only about the basic concepts.

An extension of the LL approach to metals is based on the use of the smearing techniques introduced in the static case for lattice-dynamical calculations [30. In metals, electronic levels crossing the Fermi level do not have integer occupancy (0 or 1), thus smearing techniques must be used to describe the electronic states and allow them to have partial occupancy (between 0 and 1 ). The LL approach can still have the same form as for non-metallic systems but the following modifications: i) the projector on empty states, $\hat{\mathrm{P}}_{c}^{\mathbf{k}+\mathbf{q}}$, is redefined in a different way, and ii) a fractional occupancy of electronic states should be allowed for, by introducing a suitable weight in sums over $\mathbf{k}$ points and band indices. An interested reader can find a detailed discussion in the indicated references.

The CPU time and memory requirements of the LL calculation can be very significantly reduced by employing a symmetry of the system. The space group of the crystal contains such operations as lattice translations and rotations that leave the crystal unchanged. By using these symmetry operations it is possible to reduce the number of $\mathbf{k}$ points and use only those which are in the irreducible wedge of BZ. However, in the case of EEL/IXS the symmetry of the system is reduced by the external monochromatic perturbation with a wave vector $\mathbf{Q}$. This fact must be taken into account, and thus one can consider $\mathbf{k}$ points inequivalent to each other according to the small group of $\mathbf{Q}$. 
For heavy-atom elements relativistic effects become important and thus must be taken into account for an accurate description of the EEL/IXS spectra. The LL approach has been extended to a treatment of the spin-orbit coupling effect, in a self-consistent way. In this case, instead of the scalar KS orbitals there are two-component spinors, and hence the number of electronic states in the calculation is doubled. Instead of solving non-relativistic linear-response KS equations for scalar KS orbitals in combination with non- or semi-relativistic pseudopotentials, one has to solve Pauli-type linear-response KS equations for spinors and use fully relativistic pseudopotentials. An example of the application of the relativistic LL approach is semimetallic bismuth [11, 31].

\section{Description of software components}

The turboEELS code is designed as a module of the QUANTUM ESPRESSO distribution [19]. It resides in a self contained directory TDDFPT under the root directory of the QUANTUM ESPRESSO tree, which contains also the turboTDDFT code in its two flavors (Lanczos and Davidson [17) for the calculation of the absorption spectra. The turboEELS code is tightly integrated in the turboTDDFT code and uses many of its routines. When the turboEELS code is installed (see Sec. 4.1), the bin/ directory in the QUANTUM ESPRESSO root contains links to the executables turbo_eels.x (the main program) and turbo_spectrum.x (a post-processing tool). The code turbo_eels.x performs a Lanczos recursion to obtain Lanczos coefficients $\beta_{i}$ and $\gamma_{i}$, and $z_{i}$ coefficients (see Sec. 2.5), and to thus construct a tridiagonal matrix ${ }^{M} T_{\mathbf{q}}$, while turbo_spectrum.x uses this matrix to calculate the charge-density susceptibility, $\chi(\mathbf{Q}, \mathbf{Q} ; \omega)$, according to Eqs. 32 and 33 .

\subsection{Ground-state calculation}

In order to compute the EEL/IXS spectra of a system, a standard groundstate DFT calculation has to be performed first, yielding the KS orbitals, $\mathrm{u}_{n, \mathbf{k}}^{\circ}(\mathbf{r})$, energies, $\varepsilon_{n, \mathbf{k}}^{\circ}$, for all occupied levels, and the ground-state chargedensity, $\mathrm{n}^{\circ}(\mathbf{r})$. The information thus obtained is then used as input for the linear-response calculation with the turboEELS code. This ground-state calculation is performed by the pw.x code, which is one of the key components of the QUANTUM ESPRESSO package. In Appendix B a sample input file for pw. $\mathrm{x}$ is shown for the case of diamond. After successful completion of the ground-state calculation, the pw.x code writes the ground-state KS orbitals, energies, and charge-density to disk, together with all relevant information about the system, like geometry, pseudopotentials, etc. This data is used by the turboEELS code which reads all this data at program start. Therefore, it is not necessary to redefine the system under study in the input file of turbo_eels.x.

\subsection{Linear-response TDDFT calculation}

The linear-response calculation is done using the turbo_eels. $\mathrm{x}$ code, which performs the Lanczos recursion of Sec. 2.5 for a given transferred momentum, 
Q. This is by far the most time consuming step of the calculation. In Appendix $\mathrm{B}$ a sample input file for turbo_eels. $\mathrm{x}$ is shown for the case of diamond. A list of all input variables of turbo_eels. $x$ is given in Table A.1 of Appendix A. The integer input variable itermax sets up the number of Lanczos iterations, and so determines the dimension $M$ of the tridiagonal matrix, ${ }^{M} T_{\mathbf{q}}$ (see Sec. 2.5). In fact, one can check whether the number of iterations is sufficient to achieve an adequately converged spectrum only at the post-processing level (see Sec. 3.3). It is possible to add more iterations to an existing calculation by restarting the turbo_eels.x code, setting the parameter restart=.true. and increasing itermax. The strings defined in the input variables prefix and outdir identify the system data on disk and must correspond to files created by the pw. $\mathrm{x}$ code.

The input variables $\mathrm{q} 1, \mathrm{q} 2$, and $\mathrm{q} 3$ are the three Cartesian components of the transferred momentum, $\mathbf{Q}$, specified in units of $2 \pi / a$, where $a$ is the lattice parameter specified in the ground-state calculation by pw.x. For each $\mathbf{Q}$ a separate Lanczos recursion is needed.

The calculation of the charge-density susceptibility, $\chi(\mathbf{Q}, \mathbf{Q} ; \omega)$, which is used to obtain the loss function, $-\operatorname{Im}\left[\epsilon^{-1}(\mathbf{Q}, \omega)\right]$, can be performed at different levels of theory, which are specified in the input using the parameter approximation. This parameter can take the following values: i) "TDDFT" - Time-Dependent Local Density Approximation (TDLDA) or Time-Dependent Generalized Gradient Approximation (TDGGA), depending on the XC functional; ii) "IPA" - the independent particle approximation, which implies neglecting the interaction superoperator, $\hat{\mathcal{K}}_{\mathbf{q}}$, in Eq. (24) (no Hartree and XC terms); and iii) "RPA_with_CLFE" - Random Phase Approximation (RPA) with Crystal Local Field Effects (CLFE) (describing an inhomogeneity of the system), which implies neglecting the response $\mathrm{XC}$ potential and leaving only the response Hartree potential.

One can choose which flavor of the Lanczos algorithm to use (see Sec. 2.5). By setting pseudo_hermitian=.true., the pseudo-Hermitian Lanczos algorithm will be used, otherwise the non-Hermitian Lanczos biorthogonalization algorithm will be used. It is recommended to use the former, because it is two times faster.

During the execution of the turbo_eels.x code, a file named prefix.beta_gamma_z.dat will be written to the outdir directory. This file contains the Lanczos coefficients $\beta_{i}$ and $\gamma_{i}$, and $z_{i}$ coefficients needed for the post-processing calculation. One can use this information for the analysis of the behavior of these coefficients.

\subsection{Post-processing spectrum calculation}

Once the tridiagonal matrix, ${ }^{M} T_{\mathbf{q}}$, is constructed from the Lanczos coefficients, one can compute the charge-density susceptibility according to Eqs. (32) and (33). This task is performed by the turbo_spectrum. $\mathrm{x}$ program as a postprocessing step, which requires negligible amount of the CPU time with respect to turbo_eels.x. This is so because solving a linear matrix equation $(32)$ and computing a scalar product (33) are two fast operations, which can be performed efficiently using BLAS and LAPACK libraries. 
The code turbo_spectrum. $\mathrm{x}$ is used also for the calculation of the absorption spectra computed with turboTDDFT. In order to distinguish the different applications, it is necessary to set eels=.true. in the input for turbo_spectrum.x. The labels prefix and outdir identify the system on disk and must correspond to files created by the turbo_eels. $\mathrm{x}$ code.

In Appendix B a sample input file for turbo_spectrum. $\mathrm{x}$ is shown for the case of diamond. A list of input variables for the turbo_spectrum.x program is given in Table A.2 of Appendix A.

In practice, when solving Eq. (32), a small imaginary part $\eta$ is added to the frequency argument, $\omega \rightarrow \omega+i \eta$, so as to regularize the charge-density susceptibility $\chi(\mathbf{Q}, \mathbf{Q} ; \omega)[13,25$. Setting $\eta$ to a non-zero value amounts to broadening each individual spectral line or, alternatively, to convoluting the charge-density susceptibility with a Lorentzian. The parameter $\eta$ is defined with the keyword epsil. The EEL/IXS spectra can be computed in any energy range specified by the keywords start and end, with a step of energy given by the increment parameter, all this being specified in Rydberg (units=0) or electronvolt (units $=1$ ) units.

The convergence of the spectrum in the desired energy range can be checked by varying the number of Lanczos coefficients used. This number is set by the input keywords itermax0 and itermax. If no extrapolation of Lanczos coefficients is used (extrapolation='no'), then itermax=itermax0. These variables can take values up to the number of iterations which have been calculated with the turbo_eels.x code. For a given number of Lanczos iterations, it is possible to improve the convergence of the computed spectra by extrapolating the coefficients [16]. Such an extrapolation can either be bi-constant (extrapolation='osc') or constant (extrapolation='constant') [16. In this case, the input variable itermax 0 indicates the number of exact coefficients to be read from file, while itermax is set to a value which can be chosen arbitrarily large without any significant computational cost. Such an extrapolation procedure amounts to increasing the dimension of the tridiagonal matrix, ${ }^{M} T_{\mathbf{q}}$.

The turbo_spectrum.x program generates two files, namely, prefix.plot_chi.dat which contains real and imaginary parts of the chargedensity susceptibility, $\chi(\mathbf{Q}, \mathbf{Q} ; \omega)$, for each value of the frequency $\omega$, and prefix.plot_eps.dat which contains real and imaginary parts of the dielectric function and its inverse (see Sec. 2.1).

The turbo_spectrum. $\mathrm{x}$ program will check the $f$-sum rule according to Eq. (6). However, in order to obtain a meaningful result, a convergence with respect to the frequency range of integration must be checked by the start and end parameters (the former being equal to zero, and the later being increased systematically until the convergence). In the LL approach to TDDFpT, the $f$-sum rule is satisfied exactly for any number of Lanczos iterations when local pseudopotentials are used [25]. However, a violation of the $f$-sum rule is present when non-local pseudopotentials are used. 


\section{Installation instructions and parallelization of the code}

\subsection{Installation instructions}

The turboEELS program is distributed as source code, like the other components of the QUANTUM ESPRESSO distribution. The installation procedure is the same for all modules in the QUANTUM ESPRESSO package. QuANTUM ESPRESSO and turboEELS make use of GNU autotools. The TDDFPT repository, which contains the source turboEELS code (and turboTDDFT code), must be residing within the QUANTUM ESPRESSO tree. The code is compiled with the following commands from within the QUANTUM ESPRESSO tree:

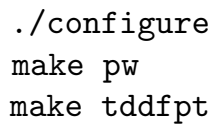

Here, the first step sets up the environment (compilers, libraries, etc.). The second step compiles the pw.x code and creates a link to this executable in the bin/ repository of the QUANTUM ESPRESSO tree. In the third step, the turboEELS codes (turbo_eels. $\mathrm{x}$ and turbo_spectrum.x) are compiled, together with the turboTDDFT codes (turbo_lanczos.x and turbo_davidson.x). Links to all these executables are created in the bin/ directory of the QUANTUM ESPRESSO tree. Further detailed installation instructions can be found in the documentation that comes with the QUANTUM ESPRESSO distribution.

The turboEELS code is tightly bound to the pw. $x$ code (residing in PW), to the ph. $\mathrm{x}$ code (residing in PHonon), and to the turboTDDFT code.

\subsection{Parallelization}

Like the other components of the QUANTUM ESPRESSO package, the turboEELS code is optimized to run on a variety of different platforms, from laptops to massively parallel architectures. The parallelization of the turboEELS code is achieved by using the message-passing paradigm and calls to standard Message Passing Interface (MPI) libraries [32]. High performance on massively parallel architectures is achieved by distributing both data and computations in a hierarchical way across processors. The turboEELS code supports two levels of parallelization: i) a plane-wave parallelization, which is implemented by distributing real- and reciprocal-space grids across the processors, and ii) a $\mathbf{k}$ points parallelization, which is implemented by dividing all processors into pools, each taking care of one or more k points. The Fast Fourier Transforms (FFT's), which are used for transformations from real space to reciprocal space and vice versa, are also efficiently parallelized among processors.

\section{Benchmarking}

We now proceed to the validation of the turboEELS code by calculating the loss function in bulk diamond. We will make a comparison with Ref. 33, where a theoretical study (using the conventional TDDFT approach) is confronted 


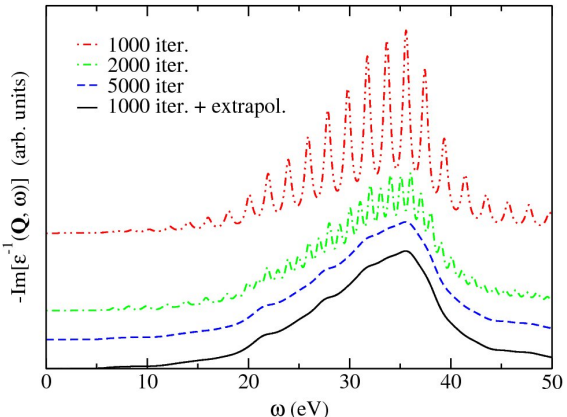

(a)

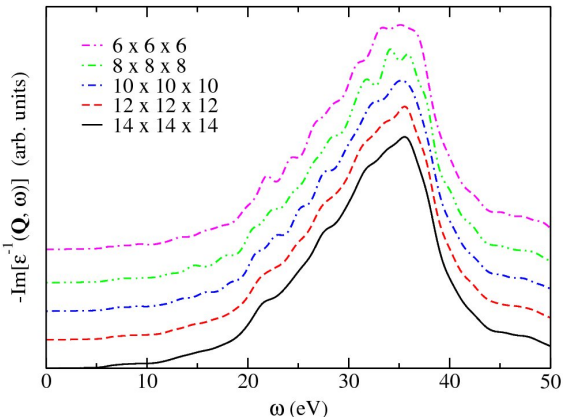

(b)

Figure 1: Loss function of diamond at $Q=0.15 \AA^{-1}$ along the [100] direction. (a) Convergence with respect to the number of Lanczos iterations, and effect of the extrapolation technique, using a $14 \times 14 \times 14$ Monkhorst-Pack $\mathbf{k}$ point mesh. (b) Convergence with respect to the size of the $\mathbf{k}$ point mesh, for 1000 Lanczos iterations using extrapolation technique. Curves have been shifted vertically for clarity.

with the experimental EEL spectra. Our purpose here is not to analyze the EEL spectra of diamond or to achieve a remarkable agreement with experiment, but to demonstrate the correctness of our implementation of turboEELS by comparing the computed EEL spectrum with the one obtained using a conventional TDDFT approach, and to show the convergence behavior of the EEL spectra when using the LL approach to EEL/IXS.

We have chosen the parameters of our calculations as close as possible to those of Ref. [33] in order to simplify the comparison. We have used the experimental lattice parameter of $3.57 \AA$ [34, and used the local density approximation (LDA) with the Perdew-Zunger parametrization of the electron-gas data [35. A norm-conserving pseudopotential was employed from the QUANTUM ESPRESSO database [36, and we have used a kinetic-energy cutoff of 50 Ry. The first BZ has been sampled with a $14 \times 14 \times 14$ Monkhorst-Pack (MP) kpoint mesh [37, resulting in $280 \mathrm{k}$-points in the irreducible wedge of BZ for the ground-state calculation, and 2940 k-points in the irreducible wedge of BZ for the small group of $\mathbf{Q}$ for the linear-response TDDFT calculation. We have used a Lorentzian broadening of $\eta=0.03$ Ry for the charge-density susceptibility (loss function). All these parameters must be specified in the following way as an input for the calculation, see Appendix B. All the results were obtained at the TDLDA level, i.e. inlcuding CLFE and XC effects.

Figure 1 shows the convergence of the loss function of diamond with respect to the number of Lanczos iterations and k points in the BZ, for $Q=0.15 \AA^{-1}$ along the [100] direction. In Fig. 1(a) it can be seen that after 1000 Lanczos iterations the loss function shows spurious wiggles, which disappear by increasing the number of Lanczos iterations up to 5000. However, the speed of the convergence can be increased by using the extrapolation technique [13, 16]. Indeed, 


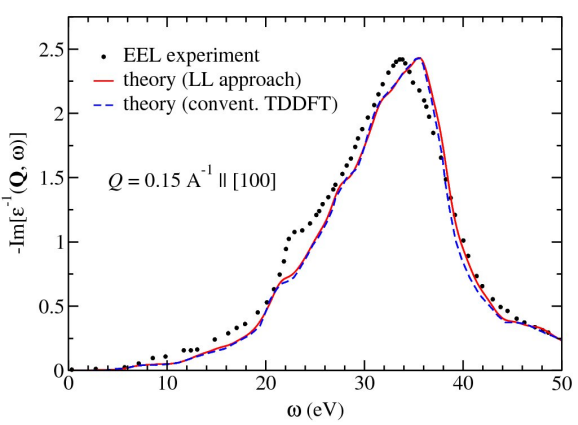

(a)

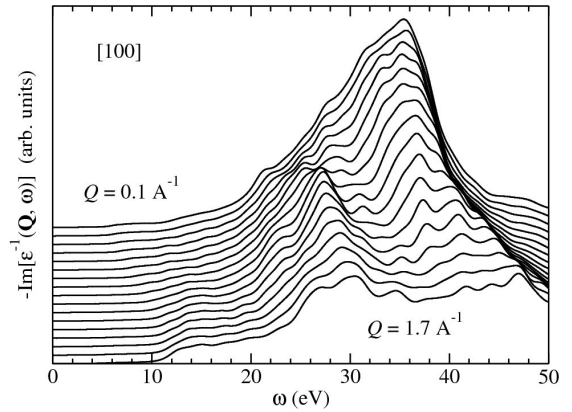

(b)

Figure 2: (a) Comparison of the loss function of diamond for $Q=0.15 \AA^{-1}$ along the [100] direction calculated using the Liouville-Lanczos (LL) approach, with experiment and with previous calculations [33]. (b) Loss function calculated using the LL approach for various transferred momenta $Q$ along [100] ranging from $0.1 \AA^{-1}$ to $1.7 \AA^{-1}$ with a step $0.1 \AA^{-1}$.

convergence can be reached after 1000 Lanczos iterations when the extrapolation of Lanczos coefficients is used (up to 20000 in this case). In Fig. 1(b) one can see that the convergence of the loss function with respect to the number of $\mathbf{k}$ points (size of the MP mesh) is reached for the $14 \times 14 \times 14 \mathrm{MP}$ mesh.

In Fig. 2 we compare our result obtained using the LL approach to EEL/IXS with those obtained using the conventional TDDFT approach based on the solution of the Dyson-like equation and with experiment [33], for $Q=0.15 \AA^{-1}$ along the [100] direction. The agreement between the LL approach and previous theoretical calculations is excellent, which validates the LL approach. It has the same level of accuracy as the conventional TDDFT approach, but requires much smaller computational cost. The agreement between theory and experiment is remarkable, though there are some small deviations. In Fig. 2(a), the plasmon peak is around at $35 \mathrm{eV}$, the peak due to interband transitions is around $22 \mathrm{eV}$. The shoulders at $28 \mathrm{eV}$ and $32 \mathrm{eV}$ are not seen in the experimental spectrum due to the large broadening, but are resolved in the theoretical spectrum. Figure 2(b) shows the loss function of diamond for various transferred momenta $Q$ along the [100] direction ranging from $0.1 \AA^{-1}$ to $1.7 \AA^{-1}$ with a step $0.1 \AA^{-1}$. One can see a strong dispersion of the plasmon peak and its damping due to entrance in the electron-hole continuum, a weak dispersion of the peak at $22 \mathrm{eV}$ due to interband transitions and its fast damping, and an appearance of the new peak at $Q=0.8 \AA^{-1}$ around at $26 \mathrm{eV}$ which also shows a weak dispersion. A detailed analysis of the loss function of diamond and its dispersion along various directions can be found in Ref. 33.

From the knowledge of the real and imaginary parts of the inverse dielectric function for a given transferred momentum, $\epsilon^{-1}(\mathbf{Q}, \omega)$, we have computed the real and imaginary parts of the dielectric function, $\epsilon(\mathbf{Q}, \omega)$, as explained in Sec. 2.1. The result is illustrated in Fig. 3 As can be seen, the plasmon at 


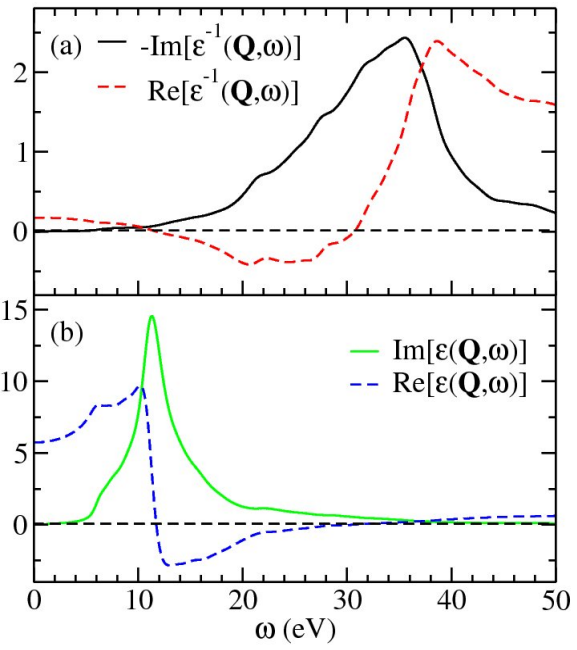

Figure 3: Real and imaginary parts of the inverse dielectric function (a) and the dielectric function (b) for $Q=0.15 \AA^{-1}$ along the [100] direction calculated using the Liouville-Lanczos (LL) approach.

$\sim 35 \mathrm{eV}$ occurs when $\operatorname{Re}[\epsilon(\mathbf{Q}, \omega)]$ is almost zero and changes its sign with a positive slope, and $\operatorname{Im}[\epsilon(\mathbf{Q}, \omega)]$ is very small. The strongest peak in $\operatorname{Im}[\epsilon(\mathbf{Q}, \omega)]$ occurs at $\sim 11 \mathrm{eV}$, which is due to interband transitions, and occuring when $\operatorname{Re}[\epsilon(\mathbf{Q}, \omega)]=0$ and changes its sign with a negative slope. And the peak in the loss function $-\operatorname{Im}\left[\epsilon^{-1}(\mathbf{Q}, \omega)\right]$ at $\sim 22 \mathrm{eV}$ is indeed due to interband transitions, because there is also a weak peak in $\operatorname{Im}[\epsilon(\mathbf{Q}, \omega)]$ around the same frequency. A detailed discussion about the origin of the peaks in the EEL spectrum of diamond can be found in Ref. [33].

Finally, we have checked the $f$-sum rule according to Eq. (6). We have found that it is satisfied with an extremely small violation of $<1 \%$, which is due to a non-locality of the pseudopotential used in our calculations [25].

\section{Conclusions}

We have presented the turboEELS code that implements a Liouville-Lanczos approach to time-dependent density functional perturbation theory for the computation of EEL and IXS spectra for any finite transferred momentum $\mathbf{Q}$. We have presented a theoretical description of the approach and its implementation as part of the QUANTUM ESPRESSO package.

The turboEELS code has a numerical scalability of the same order as standard ground-state DFT calculations. It does not require the calculation of empty electronic states, and a computationally expensive inversion of the dielectric matrix is replaced by a very efficient recursive Lanczos algorithm, which allows us to compute EEL/IXS spectra in a wide energy range. These advances allow 
us to compute EEL/IXS spectra of complex systems, e.g. high-Miller-index surfaces.

In the same spirit as the QUANTUM ESPRESSO project, turboEELS provides scientists worldwide with a well commented and open-source framework for implementing their ideas. It is in our best hope that turboEELS can benefit from the already well established users community of QUANTUM ESPRESSO for incorporating new ideas and keep growing in the future. The turboEELS code is hosted in a community accessible SVN repository [38] and hence, apart from releases in QUANTUM ESPRESSO, those who are willing to test the latest experimental implementations are welcome to do so and to contribute with their feedback.

The turboEELS code can be extended so as to employ hybrid XC functionals (which are already supported by the turboTDDFT code at $\mathbf{k}=0$ [17]), which would allow us to describe excitons and their dispersion [39.

\section{Acknowledgements}

We thank S. de Gironcoli, A. Dal Corso, M. Saitta, L. Reining, and T. Gorni for valuable discussions. The work of I.T. and N.V. has been performed under the auspices of the Laboratoire d'excellence en nanosciences et nanotechnologies Labex Nanosaclay. Support from the ANR (Project PNANO ACCATTONE) and from DGA are gratefully acknowledged. Computer resources were provided by GENCI (Project No. 2210) and CINECA (Project ISCRA C "t-EELS_E”). Technical support from C. Cavazzoni and F. Affinito at CINECA is gratefully acknowledged. 


\section{Appendix A. Input Variables}

\begin{tabular}{|c|c|c|c|}
\hline Card & Variable name & $\begin{array}{c}\text { Default } \\
\text { Value }\end{array}$ & Description \\
\hline 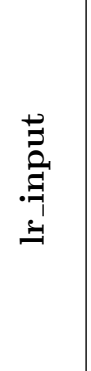 & $\begin{array}{l}\text { prefix } \\
\text { outdir } \\
\text { restart } \\
\text { restart_step } \\
\text { lr_verbosity }\end{array}$ & $\begin{array}{l}\text { 'pwscf' } \\
\quad . / \text {, } \\
\text { false. } \\
\text { itermax } \\
\quad 1\end{array}$ & $\begin{array}{l}\text { The files generated by the ground state pw.x run should have this } \\
\text { same prefix. } \\
\text { Working directory. On start, it should contain the files generated by } \\
\text { a ground state pw.x run. } \\
\text { When set to .true., turbo_eels.x will attempt to restart from a pre- } \\
\text { vious interrupted calculation. (see restart_step variable). } \\
\text { The code writes restart files every restart_step iterations. Restart } \\
\text { files are automatically written at the end of itermax Lanczos steps. } \\
\text { Verbosity level }\end{array}$ \\
\hline 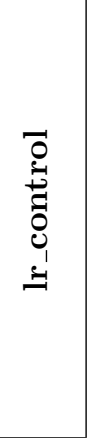 & $\begin{array}{l}\text { itermax } \\
\text { q1, q2, q3 }\end{array}$ & $\begin{array}{c}500 \\
1,1,1\end{array}$ & $\begin{array}{l}\text { Number of iterations to be performed. } \\
\text { Cartesian components of the transferred momentum in units of } 2 \pi / a \\
\text { (where } a \text { is the lattice parameter of the unit cell). } \\
\text { A string describing an approximation: 'TDDFT' - Time-Dependent Lo- } \\
\text { cal Density Approximation or Time-Dependent Generalized Gradient } \\
\text { Approximation (depending on the XC functional used), 'IPA' - In- } \\
\text { dependent Particle Approximation, 'RPA_with_CLFE' - Random Phase } \\
\text { Approximation with Crystal Local Field Effects. } \\
\text { If .true. then the pseudo-Hermitian Lanczos algorithm is used, if } \\
\text { false. then the non-Hermitian Lanczos biorthogonalization algorithm } \\
\text { is used (which is two times slower). }\end{array}$ \\
\hline
\end{tabular}

Table A.1: Input variables for turbo_eels.x 


\begin{tabular}{|c|c|c|c|}
\hline Card & Variable name & $\begin{array}{c}\text { Default } \\
\text { Value }\end{array}$ & Description \\
\hline & $\begin{array}{l}\text { prefix } \\
\text { outdir } \\
\text { eels } \\
\text { itermax0 } \\
\text { itermax } \\
\text { extrapolation } \\
\text { epsil } \\
\text { units } \\
\text { start } \\
\text { end } \\
\text { increment } \\
\text { verbosity }\end{array}$ & $\begin{array}{c}\text { 'pwscf' } \\
\cdot / \\
\text { false. } \\
1000 \\
1000\end{array}$ & $\begin{array}{l}\text { Prefix of the files generated by the previous turbo_eels.x run. } \\
\text { The directory where the output files produced by the previous } \\
\text { turbo_eels.x run are stored. } \\
\text { Must be set to .true. for EELS. EELS-specific operations will be } \\
\text { performed. } \\
\text { Number of Lanczos coefficients to be read from the file. } \\
\text { The total number of Lanczos coefficients that will be considered in } \\
\text { the calculation of the charge-density susceptibility (loss function). If } \\
\text { itermax > itermax0, the Lanczos coefficients in between itermax0+1 } \\
\text { and itermax will be extrapolated. } \\
\text { Sets the extrapolation scheme for Lanczos coefficients. 'osc' = bi- } \\
\text { constant extrapolation; 'constant' = constant extrapolation; 'no' = } \\
\text { no extrapolation. } \\
\text { The Lorentzian broadening parameter (in Rydberg units). } \\
\text { Unit system used. 0: Rydbergs; } 1 \text { : Electronvolts } \\
\text { The charge-density susceptibility and the loss function are computed } \\
\text { starting from this value. In units set by the units variable. } \\
\text { The charge-density susceptibility and the loss function are computed } \\
\text { up to this value. In units set by the units variable. } \\
\text { Incremental step used to define the mesh between start and end. In } \\
\text { units set by the units variable. } \\
\text { This integer variable controls the output verbosity. }\end{array}$ \\
\hline
\end{tabular}

Table A.2: Input variables for turbo_spectrum.x

\section{Appendix B. Sample input files}

Input example 1: Input sample for $\mathrm{pw} . \mathrm{x}$

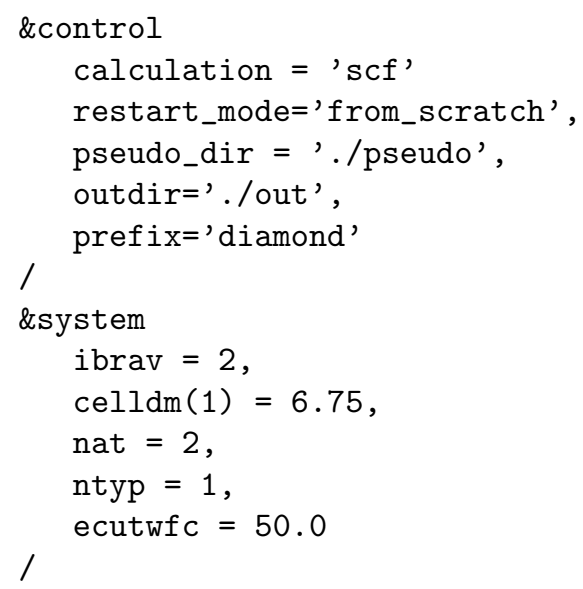




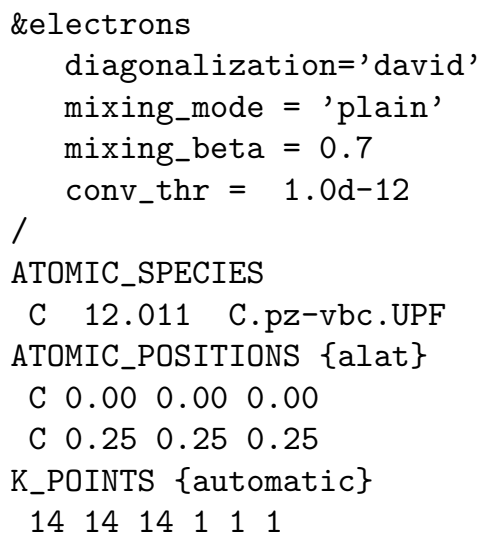

Input example 2: Input sample for turbo_eels.x

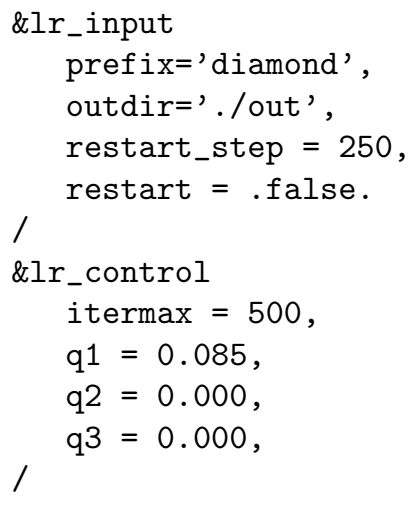

Input example 3: Input sample for turbo_spectrum.x

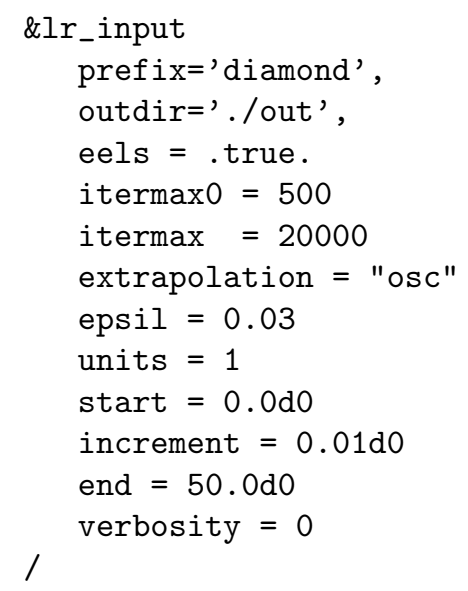




\section{References}

[1] R. F. Egerton, Electron Energy-Loss Spectroscopy in the Electron Microscope, 2nd Edition, Plenum, New York and London, 1996.

[2] W. Schülke, Electron Dynamics by Inelastic X-Ray Scattering, Oxford University Press, Oxford, 2007.

[3] S. Mazzucco, O. Séphan, C. Colliex, I. Pastoriza-Santos, L. Liz-Marzan, F. J. G. de Abajo, M. Kociak, Spatially resolved measurements of plasmonic eigenstates in complex-shaped, asymmetric nanoparticles: gold nanostars, Eur. Phys. J. Appl. Phys. 54 (2011) 33512.

[4] A. Rivacoba, N. Zabala, J. Aizpurua, Image potential in scanning transmission electron microscopy, Prog. Surf. Sci. 65 (2000) 1.

[5] F. J. G. de Abajo, M. Kociak, Probing the photonic local density of states with electron energy loss spectroscopy, Phys. Rev. Lett. 100 (2008) 106804.

[6] E. Runge, E. Gross, Density-functional theory for time-dependent systems, Phys. Rev. Lett. 52 (1984) 997.

[7] E. K. U. Gross, J. F. Dobson, M. Petersilka, Density Functional Theory of Time-Dependent Phenomena, Topics in Current Chemistry, SpringerVerlag, Berlin, 1996.

[8] M. A. L. Marques, N. T. Maitra, F. M. S. Nogueira, E. K. U. Gross, A. Rubio (Eds.), Fundamentals of Time-Dependent Density Functional Theory, Vol. 837, Lecture Notes in Physics, Springer-Verlag, Berlin Heidelbnerg, 2012.

[9] G. Onida, L. Reining, A. Rubio, Electronic excitations: Density functional versus many body Green's functions approaches, Rev. Mod. Phys. 74 (2002) 601.

[10] I. Timrov, N. Vast, R. Gebauer, S. Baroni, Electron energy loss and inelastic x-ray scattering cross sections from time-dependent density-functional perturbation theory, Phys. Rev. B 88 (2013) 064301; ibid. 91 (2015) 139901.

[11] I. Timrov, $A b$ initio study of plasmons and electron-phonon coupling in bismuth: From free-carrier absorption towards a new method for elecrton energy-loss spectroscopy, Ph.D. thesis, Ecole Polytechnique, France (2013). URL http://pastel.archives-ouvertes.fr/pastel-00823758

[12] B. Walker, R. Gebauer, Ultrasoft pseudopotentials in time-dependent density-functional theory, J. Chem. Phys. 127 (2007) 164106.

[13] D. Rocca, R. Gebauer, Y. Saas, S. Baroni, Turbo charging time-dependent density-functional theory with lanczos chains, J. Chem. Phys. 128 (2008) 154105 . 
[14] S. Baroni, P. Giannozzi, A. Testa, Green's-function approach to linear response in solids, Phys. Rev. Lett. 58 (1987) 1861.

[15] S. Baroni, S. de Gironcoli, A. D. Corso, P. Giannozzi, Phonons and related crystal properties from density-functional perturbation theory, Rev. Mod. Phys. 73 (2001) 515.

[16] O. B. Malcioglu, R. Gebauer, D. Rocca, S. Baroni, turboTDDFT - A code for the simulation of molecular spectra using the liouville-lanczos approach to time-dependent density-functional perturbation theory, Comput. Phys. Commun. 182 (2011) 1744.

[17] X. Ge, S. J. Binnie, D. Rocca, R. Gebauer, S. Baroni, turboTDDFT 2.0 - Hybrid functionals and new algorithms within time-dependent densityfunctional perturbation theory, Comput. Phys. Commun. 185 (2014) 2080.

[18] [link]. URL http://www.gnu.org/licenses/gpl.html

[19] P. Giannozzi, S. Baroni, N. Bonini, M. Calandra, R. Car, C. Cavazzoni, D. Ceresoli, G. Chiarotti, M. Cococcioni, I. Dabo, A. Dal Corso, S. De Gironcoli, S. Fabris, G. Fratesi, R. Gebauer, U. Gerstmann, C. Gougoussis, A. Kokalj, M. Lazzeri, L. Martin-Samos, N. Marzari, F. Mauri, R. Mazzarello, S. Paolini, A. Pasquarello, L. Paulatto, C. Sbraccia, S. Scandolo, G. Sclauzero, A. Seitsonen, A. Smogunov, P. Umari, R. Wentzcovitch, Quantum espresso: A modular and open-source software project for quantum simulations of materials, J. Phys.: Condens. Matter 21 (2009) 395502.

URL http://www . quantum-espresso.org

[20] S. Huotari, Spectroscopy in the Frequency Domain, Ref. [8], chapter 2, p. $15-28$.

[21] D. Pines, P. Nozières, The Theory of Quantum Liquids, Vol. 1, Benjamin, New York, 1966.

[22] R. Martin (Ed.), Electronic Structure: Basic Theory and Practical Methods, Cambridge University Press, Cambridge, 2004.

[23] R. Car, E. Tosatti, S. Baroni, S. Leelaprute, Dielectric band structure of crystals: General properties and calculations for silicon, Phys. Rev. B 24 (1981) 985.

[24] G. D. Mahan, Many-particles physics, 2nd Edition, Plenum Press, New York, 1975.

[25] S. Baroni, R. Gebauer, The Liouville-Lanczos Approach to TimeDependent Density-Functional (Perturbation) Theory, Ref. 8], chapter 19, p. $375-390$. 
[26] E. K. U. Gross, W. Kohn, Local density-functional theory of frequencydependent linear response, Phys. Rev. Lett. 55 (1985) 2850.

[27] Y. Saad, Iterative Methods for Sparse Linear Systems, 2nd Edition, SIAM, Philadelphia, 2003.

[28] M. Grüning, A. Marini, X. Gonze, Implementation and testing of lanczosbased algorithms for random-phase approximation eigenproblems, Comp. Mater. Sci. 50 (2011) 2148.

[29] A. Mostafazadeh, Pseudo-hermiticity versus pt symmetry: The necessary condition for the reality of the spectrum of a non-hermitian hamiltonian, J. Math. Phys. 43 (2002) 205.

[30] S. de Gironcoli, Lattice dynamics of metals from density-functional perturbation theory, Phys. Rev. B 51 (1995) 6773.

[31] I. Timrov, N. Vast, R. Gebauer, S. Baroni, Ab initio study of electron energy loss spectra of bulk bismuth up to $100 \mathrm{ev}$, (in preparation).

[32] Message passing interface forum, Int. J. Supercomput. Appl. 8 (1994) 159.

[33] S. Waidmann, M. Knupfer, B. Arnold, J. Fink, A. Fleszar, W. Hanke, Local-field effects and anisotropic plasmon dispersion in diamond, Phys. Rev. B 61 (2000) 10149.

[34] R. W. Wyckoff, Crystal Structures, 2nd Edition, Vol. 1, John Wiley and Sons Ltd, New York, 1963.

[35] J. Perdew, A. Zunger, Self-interaction correction to density-functional approximations for many-electron systems, Phys. Rev. B 23 (1981) 5048.

[36] The Quantum ESPRESSO pseudopotential library, http://www. quantum-espresso.org/pseudopotentials, C.pz-vbc.UPF.

[37] H. Monkhorst, J. Pack, Special points for brillouin-zone integrations, Phys. Rev. B 13 (1976) 5188.

[38] [link]. URL http://qe-forge.org/gf/project/q-e/

[39] M. Gatti, F. Sottile, Exciton dispersion from first principles, Phys. Rev. B 88 (2013) 155113. 